\title{
Clinical Efficacy of Combination Therapy for the Treatment of Severe Infections Caused by Carbapenem Resistant Gram-Negatives. A Systematic Review of Clinical Studies
}

\author{
Alessia Savoldi ( $\square$ alessia.savoldi@univr.it) \\ University of Verona: Universita degli Studi di Verona https://orcid.org/0000-0003-1873-5323

\section{Elena CARRARA} \\ University of Verona: Universita degli Studi di Verona

\section{Laura JV Piddock} \\ Global Antibiotic Research and Development Partnership
}

\section{Francois FRANCESCHI}

Global Antibiotic Research and Development Partnership

\section{Sally ELLIS}

Global Antibiotic Research and Development Partnership

\section{Margherita CHIAMENTI}

University of Verona: Universita degli Studi di Verona

\section{Damiano BRAGANTINI}

University of Verona: Universita degli Studi di Verona

\section{Elda RIGHI}

University of Verona: Universita degli Studi di Verona

\section{Evelina TACCONELLI}

University of Verona: Universita degli Studi di Verona

\section{Research article}

Keywords: carbapenem resistant Gram negative, systematic review, combination of antibiotics, antibiotic treatment

Posted Date: March 31st, 2021

DOl: https://doi.org/10.21203/rs.3.rs-366344/v1

License: (9) This work is licensed under a Creative Commons Attribution 4.0 International License. Read Full License 


\section{Abstract}

Background: The combination of antibiotics has become the clinicians' preferred strategy for the treatment of sepsis due to carbapenem-resistant Gram-negative bacteria (CR-GNB) although the evidence supporting its use is weak. This systematic review aimed at critically evaluating all available antibiotic options for CR-GNB sepsis with particular focus on combination.

Methods: Comparative and non-comparative observational studies, randomized control trials (RCTs) and case series were considered eligible with no language restriction. Studies were included if reporting microbiologically-confirmed sepsis caused by Acinetobacter baumannii, Enterobacterales/Klebsiella spp., or Pseudomonas aeruginosa, reporting at least one of the study outcome and targeted antibiotic treatment. Carbapenem-resistance was defined as phenotypically-detected in vitro resistance to at least one of the following carbapenems: doripenem, ertapenem, imipenem, meropenem. Primary outcomes were 30-day and attributable mortality. Bayesian network meta-analysis (NMA) approach was selected to explore feasibility of pooling data on antibiotic regimens.

Results: A total of 6306 records were retrieved and 134 studies (11546 patients) were included [54 studies on Acinetobacter, 52 on Enterobacterales/Klebsiella, 21 on mixed Gram-negative, and 7 on Pseudomonas]. Nine (7\%) were RCTs; 19 prospective cohorts (14\%), 89 (66\%) retrospective and 17 (13\%) case series. Forty-one studies (31\%) were multicentric. Ninety-two distinct regimens were identified with 47 (51\%, 5863 patients) not reporting any details on combination. 30-day mortality was assessed in 65 $(48 \%)$ studies while attributable mortality was reported only in $19(14 \%)$. The NMAs were not applicable for any of selected outcomes given the presence of too many disconnected components.

Conclusion: The existing evidence is too weak for allowing formulating any evidence-based therapeutic recommendation for combination of antibiotics to treat CR-GNB sepsis. Future studies must clearly define number, type, dosages, and in vitro activity of each antibiotic included in the regimen to drive recommendations for using combination of antibiotics that might be reliably applied into clinical practice.

\section{Introduction}

Carbapenem-resistant Gram-negative bacteria (CR-GNB) can cause a range of serious infections in hospitalised patients, requiring the prompt initiation of antibiotic treatment. ${ }^{1}$ Currently, the availability of active antibiotic options is very limited because of the relentless increase of extensively drug-resistant (XDR) and pan drug-resistant (PDR) strains. ${ }^{2,3}$ In recent years, only a few new agents have been granted approval for the treatment of CR-GNB infections; however, evidence on their efficacy against specific bacterial phenotypes is still scarce. Additionally, none of these new agents belongs to new antibiotic classes and the development of resistance has been reported after clinical use. ${ }^{4,5}$

The severity of those clinical scenarios, combined with the constant uncertainty around antibiotics' efficacy in terms of clinical and microbiological outcomes, has led clinicians to use combinations of 
multiple agents as a preferred strategy for treating CR-GNB infections in clinical practice. There are only a handful of guidelines supporting this choice and they are based on low-quality evidence. ${ }^{6,7}$ Moreover, due to the inadequacy of available studies, recommendations are usually generic without clearly specifying type and number of agents that should be used in combination. ${ }^{8,9}$ As a consequence, clinicians are prescribing a wide range of treatment regimens in their current practice with no proven efficacy and potential side effects from both patient- and stewardship-perspectives.

This study was conducted as part of the COHERENCE project, a Global Antibiotic Research \& Development Partnership (GARDP) initiative aimed at summarizing evidence and common practice relative to the use of combination therapy for treating severe infections caused by CR-GNB. We report the results of the systematic review of the literature on available antibiotic options for the treatment of infections caused by CR-GNB (Acinetobacter, Pseudomonas and Enterobacterales).

\section{Methods}

\section{Study selection and eligibility criteria}

Studies on adult and paediatric populations with sepsis caused by CR-GNB (Acinetobacter baumannii., Enterobacterales, Pseudomonas aeruginosa) receiving any type of antibiotic treatment after results of in vitro susceptibility testing were included. Comparative and non-comparative observational studies, randomized control trials (RCTs) and case series were considered eligible. Inclusion criteria were: studies reporting microbiologically-confirmed infections; studies reporting outcomes on CR-GNB without any further classification or on at least one of the following CR bacteria: A. baumannii, Enterobacterales/Klebsiella spp., P. aeruginosa; studies reporting data on targeted antibiotic treatment. Exclusion criteria were: studies reporting data exclusively on empiric treatment; studies reporting data on suspected infections without microbiological confirmation; studies not reporting carbapenem-resistance confirmed by phenotypic result; studies focusing only on colonization; studies reporting data on nonbacteraemic urinary tract infection; case reports, and editorials.

\section{Definitions and outcomes}

Carbapenem-resistance was defined as phenotypically-detected in vitro resistance of the isolate to at least one of the following carbapenems: doripenem, ertapenem, imipenem, meropenem. The primary outcomes were 30-day all-cause mortality and attributable mortality. If 30-day mortality was not available, all-cause mortality at any time-point was extracted. Secondary outcomes were: clinical cure, microbiological cure, length of hospital stay (LOS), re-hospitalization, resistance development after antibiotic treatment and any adverse events. Attributable mortality was defined as death due to CR-GNB infection as reported by the study. Re-hospitalization was defined as new hospital admission due to infection within 30-90 days from the initial episode. With regard to the definitions of clinical cure, microbiological cure, LOS, and costs, the definition provided by each study was accepted. Underlying comorbidities were defined as follows: pulmonary disease, including chronic pneumopathy and/or 
chronic obstructive pulmonary disease; neurological disease, including any acute or chronic neurological condition; renal disease, including any degree of acute/chronic renal impairment, and/or ongoing dialysis. The definition of immunocompromised state was fulfilled by the presence of one of the following conditions: long-term steroid therapy, haematological malignancy, neutropenic state. Each antibiotic regimen was classified as "defined antibiotic regimen" when at least the antibiotic classes (i.e. carbapenem plus polymyxin) composing the regimen was clearly specified.

\section{Search strategy}

A comprehensive search of peer-reviewed literature was carried out by searching the following electronic databases: PubMed, Cochrane Library, and Clinicaltrials.gov starting from the inception until December 2018. PubMed was searched by conducting three separate search strategies according to selected bacterial phenotype as follows: A. baumannii, Enterobacterales/Klebsiella spp., P. aeruginosa. The search was not restricted by language, year of publication or study design. Additionally, references to the included studies were systematically screened for the inclusion of relevant articles. The search strings are reported in Table S1.

\section{Data extraction}

Two reviewers (A.S. and E.C.) screened independently the title/abstract of all publications. Subsequently, the same reviewers examined the full-texts of all the potentially eligible records passing the first search step for eligibility. Inconsistencies and discrepancies were discussed and resolved after the achievement of agreement between the two reviewers or by involving a third reviewer (E.T). The following variable domains were entered into a pre-defined excel database: study-related variables (authors, year of publication, study country and design, setting), patient-related variables (age, sex, underlying comorbidities), infection-related variables (severity score, sepsis/septic shock, infection source) and treatment-related variables (number and type of agents, route of administration, dosage, duration).

\section{Quality appraisal}

Risk of bias (RoB) for individual studies was assessed by two independent reviewers (A.S. and E.C.) using Cochrane RoB tool for RCTs ${ }^{10}$ and an adapted version of RoB (Risk Of Bias tool for Non-randomized Studies of Interventions ROBINS-I) ${ }^{11}$ for observational studies. Discrepancies between the two reviewers were clarified by discussion or by involving a third reviewer (E.T.). For both RoB tools, each domain was assigned low, moderate, serious, unknown (if no sufficient information). Detailed description of the tools employed for assessment of the study quality is displayed in Table S2.

\section{Data synthesis and analysis}

The results of the systematic review were stratified according to the following bacterial phenotypes: $A$. baumannii, Enterobacterales, $P$. aeruginosa, and Mixed Gram-negative, in case of the individual study reported data on aggregated CR-GNB without any further classification by species. Continuous and categorical variables were reported as median and median frequency with interquartile range (IQR), respectively. Given that considerable heterogeneity among treatment schemes was expected, with very 
few studies performing a direct pairwise comparison, we planned to explore the feasibility of pooling comparative data on the various antibiotic treatment schemes using Bayesian network meta-analysis (NMA) (STATA command "network setup"). We planned to assess heterogeneity in each pairwise comparison with $\mathrm{I}^{2}$ test, and the inconsistency between direct and indirect evidence using Wald Test (within the whole network) and node-splitting (within each specific comparison). For the quantitative analysis, we included exclusively patients receiving defined antibiotic regimens. Treatment observation was adopted as the unit of analysis. All statistical analyses were carried out with STATA version 15 (Statacorp LLC, Texas, United States). The methods were based on the framework provided by the Preferred Reporting Items for 2015 Systematic Review and Meta-Analysis Protocols for NMA (PRISMANMA) checklist. ${ }^{12}$ This study was registered on the International Prospective Register of Systematic Reviews (PROSPERO) with the following trial number: CRD42019127928 dated 9 April 2019 (https://www.crd.york.ac.uk/prospero/display_record.php?RecordID=127928).

\section{Results}

\section{Study selection and characteristics}

A total of 6306 records were retrieved from the search. After applying inclusion and exclusion criteria, 134 studies were included in the review. The flowcharts of the study selection process are in Figures S1a, S1b, S1c. The list of the included studies is provided in Annex V. Overall, the 134 studies were conducted in 19 countries and involved 11546 patients (Fig. S2). Stratifying by bacterial phenotype, 54 (40\%) studies (4395, 38\% patients) reported data on $A$. baumannii, 52 (38.5\%) studies (4118, 35.5\% patients) reported data on Enterobacterales, while only seven (5\%) studies (545, 5\% patients) specifically reported data on $P$. aeruginosa. Twenty-one (16.5\%) studies (2488, $21.5 \%$ patients) reported data on unspecified mixed Gram-negative. Eighty-nine (66\%) studies had a retrospective design, whereas 19 (14\%) and $17(13 \%)$ were case series and prospective, respectively. Nine (7\%) were randomized studies. Ninety-three $(69 \%)$ studies were conducted in a single hospital, while the remaining $41(31 \%)$ studies were multicentric. With regard to the study setting, $81(61 \%)$ studies were performed across hospital wards, while the remaining 53 (39\%) were conducted in an Intensive Care Unit (ICU) setting.

\section{Patient and infection characteristics}

Adult patients. One hundred and thirty-one (98\%) studies focused on adults, including a total of 11472 hospitalized patients (4391 Acinetobacter, 4109 Enterobacterales, 545 Pseudomonas, 2488 Mixed gramnegative). The median patient age was 59.5 years (IQR 51-65) and the median Apache II score was 20 (IQR 17-23). Only 18 (14\%) studies reported all the listed underlying comorbidities. The median frequency of immunocompromised status was $26 \%$ (IQR 14-40). Sixty-seven (51\%) studies clearly defined the source of the infection. Among those, bloodstream infections were the most common (37/131 studies, $28 \%)$, followed by hospital- or ventilator acquired pneumonia (HAP/VAP) (27/131 studies, 20\%). Information on monomicrobial/polymicrobial infection was reported in 79 (60\%) studies. Overall, 35 studies (26\%) had monomicrobial infections as a specific inclusion criterion. Data on 
'appropriate empiric antibiotic treatment' was available in 31/131 studies (23\%) with a median value of $48 \%$ (IQR 22-67). Patient and infection characteristics stratified by bacterial phenotype are shown in Table 1. 
Table 1

Demographic and clinical characteristics of adult patients.

\begin{tabular}{|c|c|c|c|c|c|}
\hline \multirow{3}{*}{$\begin{array}{l}\text { Patient and infection } \\
\text { characteristics }\end{array}$} & \multicolumn{4}{|c|}{ Bacterial phenotype } & \multirow{3}{*}{$\begin{array}{l}\text { Total } \\
131 \\
\text { studies }\end{array}$} \\
\hline & $\begin{array}{l}\text { A. } \\
\text { baumannii }\end{array}$ & $\begin{array}{l}\text { P. } \\
\text { aeruginosa }\end{array}$ & \multirow{2}{*}{$\begin{array}{l}\text { Enterobacterales } \\
51 \text { studies }\end{array}$} & $\begin{array}{l}\text { Mixed Gram- } \\
\text { negative }\end{array}$ & \\
\hline & 53 studies & 7 studies & & 20 studies & \\
\hline Age years, median (IQR) & \multirow{2}{*}{$\begin{array}{l}57(51- \\
65)\end{array}$} & \multirow{2}{*}{$\begin{array}{l}59.5(46- \\
63)\end{array}$} & \multirow[t]{2}{*}{$60(51-64)$} & \multirow{2}{*}{$\begin{array}{l}59.5(51- \\
65)\end{array}$} & \multirow{2}{*}{$\begin{array}{l}59.5 \\
(51- \\
65)\end{array}$} \\
\hline $\begin{array}{l}\text { (103 studies provided } \\
\text { data) }\end{array}$ & & & & & \\
\hline \multicolumn{6}{|l|}{ Pulmonary illness } \\
\hline $\begin{array}{l}\text { Median frequency \% } \\
\text { (IQR) }\end{array}$ & $\begin{array}{l}20(10- \\
26)\end{array}$ & $\begin{array}{l}19(15- \\
28)\end{array}$ & $16(10-23)$ & $21(12-25)$ & $\begin{array}{l}20(11- \\
26)\end{array}$ \\
\hline
\end{tabular}

(64 studies provided data)

\section{Neurological illness}

Median frequency $\%$

(IQR)

$13(8-22)$

$22(9-24)$

$20(11-28)$

$17(9-$ 26)

(36 studies provided data)

\section{Renal illness}

Median frequency \% (IQR)

$23(18-$

35)

35)

$24.5(22-36)$

$20(18-33)$

$22(18-$ 35)

(76 studies provided data)

Immunocompromised status

Median frequency \% (IQR)

$26(14-$

44)

$31(15-$

41)

$29.5(18-47)$

$32(15-43)$

$26(14-$ 40)

(72 studies provided data)

Infection type, $\mathrm{n}(\%)$

Mixed infection

18 (35)

$4(57)$

$31(61)$

$11(57)$

64 (49)

Bloodstream infection

$13(24)$

0

18 (35)

$6(28.5)$

37 (28)

Ventilator-/Hospital

acquired pneumonia

20 (37)

$3(43)$

2 (4)

2 (9.5)

27 (21) 


\begin{tabular}{|c|c|c|c|c|c|}
\hline \multirow{3}{*}{$\begin{array}{l}\text { Patient and infection } \\
\text { characteristics }\end{array}$} & \multicolumn{4}{|c|}{ Bacterial phenotype } & \multirow{3}{*}{$\begin{array}{l}\text { Total } \\
131 \\
\text { studies }\end{array}$} \\
\hline & $\begin{array}{l}\text { A. } \\
\text { baumannii }\end{array}$ & $\begin{array}{l}\text { P. } \\
\text { aeruginosa }\end{array}$ & \multirow{2}{*}{$\begin{array}{l}\text { Enterobacterales } \\
51 \text { studies }\end{array}$} & $\begin{array}{l}\text { Mixed Gram- } \\
\text { negative }\end{array}$ & \\
\hline & 53 studies & 7 studies & & 20 studies & \\
\hline Other* & $2(4)$ & 0 & 0 & $1(5)$ & $3(2)$ \\
\hline \multicolumn{6}{|l|}{ Apache II score } \\
\hline Median (IQR) & \multirow[t]{2}{*}{$\begin{array}{l}18(15- \\
22)\end{array}$} & \multirow[t]{2}{*}{$\begin{array}{l}14(14- \\
16)\end{array}$} & \multirow[t]{2}{*}{$20(15-21)$} & \multirow[t]{2}{*}{$19(19-23)$} & \multirow[t]{2}{*}{$\begin{array}{l}20(17- \\
23)\end{array}$} \\
\hline $\begin{array}{l}\text { (76 studies provided } \\
\text { data) }\end{array}$ & & & & & \\
\hline Monomicrobial, n (\%) & \multirow[t]{2}{*}{$13(26)$} & \multirow[t]{2}{*}{$3(43)$} & \multirow[t]{2}{*}{$12(23)$} & \multirow[t]{2}{*}{$6(27)$} & \multirow[t]{2}{*}{$34(26)$} \\
\hline $\begin{array}{l}\text { (79 studies provided } \\
\text { data) }\end{array}$ & & & & & \\
\hline \multicolumn{6}{|l|}{$\begin{array}{l}\text { Covering empiric } \\
\text { treatment }\end{array}$} \\
\hline $\begin{array}{l}\text { Median frequency \% } \\
(\text { IQR) }\end{array}$ & \multirow{2}{*}{ - } & \multirow{2}{*}{-} & \multirow{2}{*}{-} & \multirow{2}{*}{ - } & \multirow{2}{*}{$\begin{array}{l}48(22- \\
67)\end{array}$} \\
\hline $\begin{array}{l}\text { (30 studies provided } \\
\text { data) }\end{array}$ & & & & & \\
\hline \multicolumn{6}{|c|}{$\begin{array}{l}\text { *Other: Central nervous system infections in } A \text {. baumannii group; mediastinitis in Mixed Gram- } \\
\text { negative group. }\end{array}$} \\
\hline \multicolumn{6}{|c|}{${ }^{*}$ Given that the paucity of studies providing data, only overall value was computed. } \\
\hline \multicolumn{6}{|l|}{ IQR: interquartile range } \\
\hline
\end{tabular}

Paediatric patients. Three studies (2\%) reported data on 74 paediatric patients (four on Acinetobacter, nine on Enterobacterales, 61 on Mixed Gram-negative). None of these studies reported any clinical characteristics. A summary of the studies is provided in Table S3.

\section{Microbiological characteristics}

One hundred and seven studies (80\%) specifically referred to standardized breakpoint reference systems for defining carbapenem resistance; among them, 84 studies (63\%) referred to The Clinical \& Laboratory Standards Institute and 23 studies (17\%) referred to the European Committee on Antimicrobial Susceptibility Testing. One hundred one (76\%) studies specified the microbiologic tool used for detecting carbapenem resistance. Among the six different methods employed, automated microdilution was the most common (48 studies, 36\%), followed by E-test (22 studies, 16\%) and disc diffusion (17 studies, $13 \%)$, (Table S4). The molecular mechanism causing resistance to carbapenems was specified in 39 studies (28\%), especially in the Enterobacterales group, for which the most frequent mechanism of 
resistance was the production of KPC type carbapenemase (Table S5). Quantitative data on concomitant prevalence of resistance to other antibiotic agents in CR-GNB was reported in 79/134 (58\%) studies. The highest resistance levels (above 50\%) for other antibiotic classes were frequently detected for aminoglycosides and polymyxins classes. The distribution (percentages) of the concomitant resistance by study is displayed in Figure S3.

\section{Antibiotic treatment regimens}

Overall, 92 distinct antibiotic regimens were used. Forty-five out of 92 (49\%) antibiotic regimens accounted for 5683 (49.4\%) patients were classified as "defined antibiotic regimen". Among them, 13 were single-, 21 were dual, and 11 were triple-antibiotic regimens. Polymyxin was the most prescribed antibiotic class both alone and in combination for each bacterial phenotype. Single antibiotic regimen with a polymyxin was used in 2383 patients. Nine out of 11 dual-antibiotic regimens included a polymyxin. The distribution of the antibiotic regimens grouped by patients and bacterial phenotype is detailed in Fig. 1.

The remaining 5863 (50.6\%) patients were treated with 47 (51\%) different "undefined antibiotic regimens". In this group, the definition of "antibiotic combination" was very generic and extremely heterogeneous, including a variable number and type of antibiotic agents (i.e. carbapenem-based, colistin-sparing, tigecycline-containing, any combination). For $42 / 47$ of these antibiotic combinations, the in vitro susceptibility of the included agents was unknown.

\section{Outcomes description}

The 134 studies measured seven different outcomes. The most tested outcome was mortality (118 studies, $87 \%$ ): 30-day mortality was reported in 65 (48\%) studies, while attributable mortality was reported in $19(14 \%)$ studies. Clinical cure and microbiological cure were also frequently tested by the individual studies ( 66 and 62 studies, $48 \%$ and $46 \%$ ). Full results stratified by bacterial phenotype are displayed in Table 2. 
Table 2

Outcomes assessed by the included studies clustered by bacterial phenotype

Outcome

Bacterial phenotype

Total

$\begin{array}{lllll}\text { A. } & \begin{array}{l}\text { P. } \\ \text { aeruginosa }\end{array} & \begin{array}{l}\text { Enterobacterales } \\ \text { baumannii }\end{array} & \begin{array}{l}\text { Mixed Gram- } \\ \text { negative }\end{array} & \begin{array}{l}134 \\ \text { studies }\end{array} \\ \text { 54 studies } & \mathbf{7} \text { studies } & 52 \text { studies } & 21 \text { studies } & \end{array}$

\section{Mortality}

30-day mortality

29

3

24

9

65

Mortality at other time

10

2

16

7

35

point*

Attributable mortality

11

1

3

4

19

\section{Adverse effects}

Renal

21

2

9

13

45

Diarrhea/C. difficile

infection

Neurological

8

1

2

6

Not specified

3

3

3

3

12

Clinical cure

38

6

19

13

76

Length of hospital stay 4

Microbiological cure

37

7

Resistance

development

Re-hospitalization

$4 \quad-\quad 2$

2

1

7

*Mortality at any time point other than 30 days.

\section{Quantitative synthesis}

Only the 5683 patients receiving a "defined antibiotic regimen" were included in the quantitative synthesis. Four separate NMAs were run for the outcomes mortality, clinical cure, microbiological cure and adverse events for $A$. baumannii, Enterobacterales and $P$. aeruginosa. Irrespective of the bacterial phenotype and the outcome considered, a quantitative analysis through NMA could not be performed because the network had too many disconnected components (i.e. the included regimens could be grouped in several independent components with no connections from one to the other). An example of 
the network geometry for the outcome mortality is displayed in Fig. 2. Outcome data from individual studies that were entered in the NMAs are reported in Annex III. Quantitative analyses for the remaining outcomes (LOS, re-admission, costs, resistance development and relapse of infection) were not conducted because of the scarcity of available observations (less than five for each bacterial phenotype).

\section{Quality appraisal}

With regard to non-randomized studies, 119/125 (95\%) of them had critical RoB concerning confounding, while 110/125 (88\%) and 107/125 (86\%) studies had critical RoB concerning selection of patients and classification of intervention, respectively. The majority of the domains pertaining to randomized studies were at moderate or low RoB. Table 3 summarises the quality assessment by domain. Full quality appraisal is shown in Table S6.

Table 3

Risk of Bias assessment of the studies by domain

\begin{tabular}{|c|c|c|c|c|}
\hline \multirow[t]{2}{*}{ Study type } & \multirow{2}{*}{$\begin{array}{l}\text { NON-RANDOMIZED } \\
\text { (125 studies) }\end{array}$} & \multicolumn{3}{|c|}{ Risk of bias, n (\%) } \\
\hline & & CRITICAL & MODERATE & LOW \\
\hline \multirow[t]{6}{*}{ Domain } & Confounding bias & $119(95)$ & $6(0.5)$ & 0 \\
\hline & Bias in selection of participants & $110(88)$ & $15(1.2)$ & 0 \\
\hline & Bias in classification of interventions & $107(86)$ & $18(14)$ & 0 \\
\hline & Deviation from intended interventions & $92(74)$ & $32(26)$ & $1(0.8)$ \\
\hline & Missing data & 0 & $15(12)$ & $110(88)$ \\
\hline & Bias in selection of reported results & 0 & $10(8)$ & $115(92)$ \\
\hline \multirow[t]{2}{*}{ Study type } & RANDOMIZED & CRITICAL & MODERATE & LOW \\
\hline & (9 studies) & & & \\
\hline \multirow[t]{6}{*}{ Domain } & Selection bias & $2(22)$ & $5(55)$ & $2(22)$ \\
\hline & Performance bias & $2(22)$ & $6(67)$ & $1(11)$ \\
\hline & Detection bias & $3(33)$ & $5(55)$ & $1(11)$ \\
\hline & Attrition bias & $1(11)$ & $6(67)$ & $2(22)$ \\
\hline & Reporting bias & 0 & $2(22)$ & 7 (77) \\
\hline & Other bias & 0 & $1(1)$ & $8(6)$ \\
\hline
\end{tabular}

\section{Discussion}

This systematic review provides a comprehensive and critical evaluation of the current evidence on the available antibiotic options for treating patients with sepsis due to CR A. baumannii, Enterobacterales, 
and $P$. aeruginosa. Our results clearly revealed the challenges to developing evidence-based recommendations on the therapeutic management of severe infections caused by CR-GNB from the existing literature. The included studies showed a suboptimal reporting of key-variables, making results difficult to compare and ill-suited for adjusted analysis. Despite the availability of numerical data on 11546 patients, no quantitative analyses could be performed because of the vast heterogeneity and the lack of clear definitions of the included antibiotic regimens.

The first part of our systematic review focused on a descriptive analysis of clinical and microbiological data. In patients with CR-GNB infections, especially those with critical illness, comorbidities and baseline severity of disease are known to be major contributors to the final outcomes. ${ }^{13}$ However, our systematic review showed that only 21 comparative observational studies included an adjusted analysis for these confounders, whereas the remaining studies were generally too small to allow for adjustment. Overall, the studies had a median sample of 49 , a figure which is considerably lower than the estimated sample of several hundreds or even thousands of patients that are needed for reliably assessing independent association of one antibiotic regimen with mortality in observational studies. ${ }^{6,14}$

In terms of microbiological characteristics, causative pathogens varied in terms of species, mechanisms of resistance, minimum inhibitory concentrations thresholds to carbapenems, and pattern of concomitant resistance. This heterogeneity was common not only among different studies, but often within the same study. Overall, resistance to carbapenem was detected using five microbiological methods with various performance and accuracy. ${ }^{15}$

Evidence from observational studies shows a survival benefit in patients with severe infections receiving in vitro active empirical treatment. ${ }^{16,17}$ However, a post hoc analysis of the only available RCT on the topic documented that early initiation of colistin did not impact on survival of patients with severe infections sustained by colistin-susceptible CR-GNB. ${ }^{18}$ Similarly to other clinically relevant variables, data on empirical coverage from our systematic review were scarce (only $23 \%$ of the studies) and did not allow to conduct any further analysis.

Our systematic review included mostly low-quality observational studies. The included randomized controlled trials were generally of better quality, but did not contribute significantly to the overall analysis. In particular, the trials on new antibiotics showed important limitations related to the inclusion of very small sample size and the use of heterogeneous comparison groups. ${ }^{19,20}$

In the last years, several systematic reviews and meta-analyses have addressed the issue of the superiority of combination treatment, mainly focusing on polymyxin-carbapenem combination. Conclusions from these systematic reviews were generally undermined by methodological limitations of the included studies ${ }^{6,21,22}$ and they did not match the results from a more recent RCT addressing the same clinical question. ${ }^{23}$ Our systematic review included overall 97 distinct antibiotic regimens; this result reflects the lack of standardization in clinicians' prescribing, as pointed out in the third paper of this series (reference to be added). Among these 92 regimens, less than a half were accurately defined. Most 
studies, in fact, adopted a generic definition of combination therapy frequently with unknown in vitro susceptibility, rather than defining specific combinations. In this way, patients could be easily allocated to the various treatment arms and potentially contribute to the retrospective assessment of determinants of mortality. However, in the wake of existing evidence and experts' recommendations, we deemed those data as scarcely informative for clinical practice and we restricted the analysis to clearly defined antibiotic regimens. ${ }^{21}$

The purpose of our quantitative analysis was to evaluate the effectiveness of defined antibiotic regimens in patients with sepsis due to CR A. baumannii, Enterobacterales and $P$. aeruginosa in terms of clinical and microbiological outcomes. Given that several studies did not offer a direct comparison, we selected the NMA approach that can integrate both direct evidence (from studies directly comparing two antibiotic regimens) with indirect evidence (information on two antibiotic regimens derived via a common comparator), increase the precision in the estimates and produce a relative ranking of all antibiotic regimens for selected outcomes. ${ }^{24}$ Unfortunately, the distribution of the patients across 45 defined antibiotic regimens did not allow the creation of a 'connected network' where all regimens are comparable via direct or indirect comparison. We could have forced the analysis or conducted separate NMAs for each network component, but potential results would have been again of poor clinical significance.

\section{Conclusion}

In conclusion, in our systematic review we comprehensively collated evidence on all antibiotic options for treating sepsis due to CR-GNB. Besides providing a state of art of the quantity and the quality of current literature, our study has focused on the drawbacks in the definition of "combination therapy" and the implications that it might have in clinical practice. Methodological issues and inherent biases of existing studies have precluded a quantitative synthesis of results, not allowing identification of which is the optimal therapeutic strategy, as monotherapy or combination, for treating CR A. baumannii, Enterobacterales or $P$. aeruginosa sepsis in specific clinical scenarios.

Although RCTs in this field are urgently needed to overcome many of the observed biases, they are challenging to conduct due to both operational and clinical difficulties, as recently proven by the CARE study, for example ${ }^{19}$. In this context, observational studies still play an important role if they fulfil specific requirements, such as prospective design, proper sample size, identification and adjustment for major confounders, outcome homogenization and accurate definition of the interventions. ${ }^{25,26}$ In particular, when referring to therapeutic interventions, the antibiotic regimens should be clearly specified in terms of in vitro activity, number, type, dosage and duration. ${ }^{25}$ All these measures will provide high-quality evidence-based information that finally can guide the clinical practice in the near future.

\section{Abbreviations}

CR-GNB: Carbapenem-resistant Gram-negative bacteria; RCT: randomized control trial; XDR: extensively drug-resistant; PDR: pan drug-resistant; GARDP: Global Antibiotic Research \& Development Partnership; 
LOS: length of hospital stay; RoB: Risk of Bias; ICU: intensive care unit; ROBINS-I: Risk Of Bias tool for Non-randomized Studies of Interventions; IQR: Interquartile Range; NMA: Network Meta-Analysis; PRISMA: Preferred Reporting Items Systematic Review and Meta-Analysis Protocols; HAP/VAP: hospital- and ventilator acquired pneumonia.

\section{Declarations}

\section{Ethics approval and consent to participate}

Not applicable.

\section{Consent for publication}

Not applicable.

\section{Availability of data and materials}

All data generated or analysed during this study are included in the manuscript and in its supplementary material.

\section{Competing interests}

AS, EC, LJVP, FF, SE, MC, DB, ER and ET have no competing interests to be declared.

\section{Funding}

Global Antibiotic Research and Development Partnership (GARDP) supported the entire project.

\section{Authors' contributions}

ET and LJVP conceived the idea of this paper. EC, AS, SE, FF, LJVP, MC, DB, RE and ET designed the study. AS and EC contributed to the data analysis and synthesis. AS, EC and ET wrote the paper. All authors contributed to revision of the paper and approval of the final version for submission. All authors had full access to all the data in the study and had final responsibility for the decision to submit for publication.

\section{Acknowledgements}

We would like to thank Ruth Joanna Davis for the editorial support. We would also thank Ania Gorska for the graphical support.

\section{References}

1. Isler B, Doi Y, Bonomo RA, Paterson DL. New Treatment Options against Carbapenem-Resistant Acinetobacter baumannii Infections. Antimicrob Agents Chemother 2019;63(1). DOI: 
2. Cai Y, Chai D, Wang R, Liang B, Bai N. Colistin resistance of Acinetobacter baumannii: clinical reports, mechanisms and antimicrobial strategies. J Antimicrob Chemother 2012; 67(7). DOI: $10.1093 / \mathrm{jac} / \mathrm{dks} 084$.

3. Vardakas KZ, Legakis NJ, Triarides N, Falagas ME. Susceptibility of contemporary isolates to fosfomycin: a systematic review of the literature. Int J Antimicrob Agents 2016; 47(4). DOI: 10.1016/j.ijantimicag.2016.02.001.

4. Petty LA, Henig O, Patel TS, Pogue JM, Kaye KS. Overview of meropenem-vaborbactam and newer antimicrobial agents for the treatment of carbapenem-resistant Enterobacterales. Infect Drug Resist 2018;11. DOI: 10.2147/IDR.S150447.

5. Humphries RM, Hemarajata P. Resistance to Ceftazidime-Avibactam in Klebsiella pneumoniae Due to Porin Mutations and the Increased Expression of KPC-3. Antimicrob Agents Chemother 2017;61(6). DOI: 10.1128/AAC.00537-17.

6. Paul M, Carmeli Y, Durante-Mangoni E, et al. Combination therapy for carbapenem-resistant Gramnegative bacteria. J Antimicrob Chemother 2014; 69(9). DOI: 10.1586/14787210.2013.845523.

7. Vardakas KZ, Mavroudis AD, Georgiou M, Falagas ME. Intravenous colistin combination antimicrobial treatment vs. monotherapy: a systematic review and meta-analysis. Int J Antimicrob Agents 2018; 51(4). DOI: 10.1016/j.ijantimicag.2017.12.020.

8. Hawkey PM, Warren RE, Livermore DM, et al. Treatment of infections caused by multidrug-resistant Gram-negative bacteria: report of the British Society for Antimicrobial Chemotherapy/Healthcare Infection Society/British Infection Association Joint Working Party. The Journal of antimicrobial chemotherapy 2018;73(suppl_3). DOI: 10.1093/jac/dky027.

9. Rodríguez-Baño J, Gutiérrez-Gutiérrez B, Machuca I, Pascual A. Treatment of Infections Caused by Extended-Spectrum-Beta-Lactamase-, AmpC-, and Carbapenemase-Producing Enterobacterales. Clinical microbiology reviews 2018;31(2): e00079-17. DOI: 10.1128/CMR.00079-17.

10. Higgins PJ. Savović J, Page MJ, Sterne JAC on behalf of the RoB2 Development Group. Revised Cochrane risk-of-bias tool for randomized trials (RoB 2). Available at (last access on 18 February 2020): https://drive.google.com/file/d/19R9savfPdCHC8XLz2iiMvL_71IPJERWK/view

11. Sterne JA, Hernan MA, Reeves BC, Savòvic J, Berkman ND, Viswanathan M, et al. ROBINS-I: a tool for assessing risk of bias in non-randomised studies of interventions. BMJ 2016; 355. DOI: 10.1136/bmj.i4919.

12. Hutton B, Salanti G, Caldwell DM, et al. The PRISMA extension statement for reporting of systematic reviews incorporating network meta-analyses of health care interventions: checklist and explanations. Ann Intern Med 2015;162(11). DOI: 10.7326/M14-2385.

13. Cheng IL, Chen YH, Lai CC, Tang HJ. Intravenous Colistin Monotherapy versus Combination Therapy against Carbapenem-Resistant Gram-Negative Bacteria Infections: Meta-Analysis of Randomized Controlled Trials. J Clin Med 2018;7(8):208. DOI: 10.3390/jcm7080208. 
14. Paul M, Shani V, Muchtar E, Kariv G, Robenshtok E, Leibovici L. Systematic review and meta-analysis of the efficacy of appropriate empiric antibiotic therapy for sepsis. Antimicrobial agents and chemotherapy 2010;54(11). DOI: 10.1128/AAC.00627-10.

15. Haldorsen B, Giske CG, Hansen DS, et al. Performance of the EUCAST disc diffusion method and two MIC methods in detection of Enterobacterales with reduced susceptibility to meropenem: the NordicAST CPE study. J Antimicrob Chemother 2018;73(10). DOI: 10.1093/jac/dky276.

16. Carrara E, Pfeffer I, Zusman O, Leibovici L, Paul M. Determinants of inappropriate empirical antibiotic treatment: systematic review and meta-analysis. International journal of antimicrobial agents 2018;51(4). DOI: 10.1016/j.ijantimicag.2017.12.013.

17. Du X, Xu X, Yao J, et al. Predictors of mortality in patients infected with carbapenem-resistant Acinetobacter baumannii: A systematic review and meta-analysis. Am J Infect Control 2019; 47(9). DOI: 10.1016/j.ajic.2019.03.003.

18. Zak-Doron Y, Benattar YD, Pfeffer I, et al. The Association Between Empirical Antibiotic Treatment and Mortality in Severe Infections Caused by Carbapenem-resistant Gram-negative Bacteria: A Prospective Study. Clinical infectious diseases: an official publication of the Infectious Diseases Society of America 2018;67(12).

19. A Study of Plazomicin Compared With Colistin in Patients With Infection Due to CarbapenemResistant Enterobacterales (CRE) (CARE). Available at: https://clinicaltrials.gov/ct2/show/NCT01970371 (Accessed 18 February 2020).

20. Wunderink RG, Giamarellos-Bourboulis EJ, Rahav G, et al. Effect and Safety of MeropenemVaborbactam versus Best-Available Therapy in Patients with Carbapenem-Resistant Enterobacterales Infections: The TANGO II Randomized Clinical Trial. Infect Dis Ther 2018; 7(4). DOI: 10.1007/s40121-018-0214-1.

21. Zusman O, Altunin S, Koppel F, Benattar JD, Gedik H, Paul M. Polymyxin monotherapy or in combination against carbapenem-resistant bacteria: systematic review and meta-analysis. $J$ Antimicrob Chemother 2017; 72(1). DOI: 10.1093/jac/dkw377.

22. Falagas ME, Lourida P, Poulikakos P, Rafailidis PI, Tansarli GS. Antibiotic treatment of infections due to carbapenem-resistant Enterobacterales: systematic evaluation of the available evidence. Antimicrob Agents Chemother 2014; 58(2). DOI: 10.1128/AAC.01222-13.

23. Paul M, Daikos GL, Durante-Mangoni E, et al. Colistin alone versus colistin plus meropenem for treatment of severe infections caused by carbapenem-resistant Gram-negative bacteria: an openlabel, randomised controlled trial. Lancet Infect Dis 2018; 18(4). DOI: 10.1016/S14733099(18)30099-9.

24. Chaimani A, Higgins JP, Mavridis D, Spyridonos P, Salanti G. Graphical tools for network metaanalysis in STATA. PLoS One 2013; 8(10). DOI: 10.1371/journal.pone.0076654.

25. Paul M, Scudeller L. Clinical research designs to study treatment effects for multidrug-resistant bacteria. Clin Microbiol Infect 2019; 25(8). DOI: 10.1016/j.cmi.2019.05.004. 
26. Paul M, Leibovici L. Observational studies examining patient management in infectious diseases. Clin Microbiol Infect 2017; 23(3). DOI: 10.1016/j.cmi.2016.05.005.

\section{Figures}

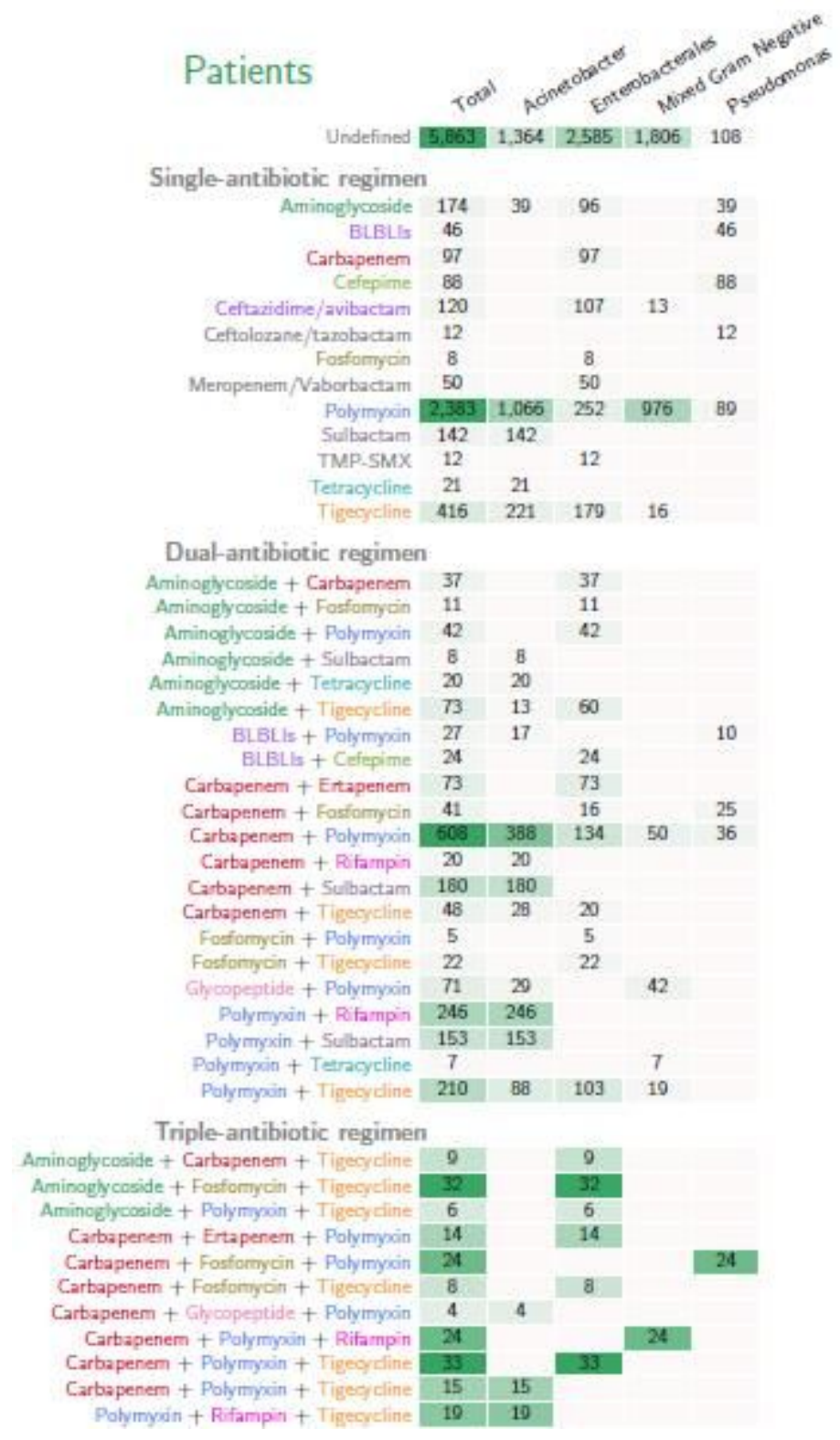

\section{Figure 1}

Antibiotic regimens assessed in the included studies stratified by bacterial phenotype and number of patients. Legend: The antibiotics belonging to same classes are grouped. Carbapenem classes includes Group A carbapenem (doripenem, imipenem, meropenem). Tigecycline is the only agent belongs to the class of glycyclyine. Sulbactam was grouped separately for Acinetobacter. The computation of patients referred to the outcome mortality (or clinical cure, if mortality was not reported by the individual study). In case of multiple outcomes, the number of patients for each antibiotic regimen was computed for only one outcome. BLBLIs: beta lactam-beta lactam inhibitors. 

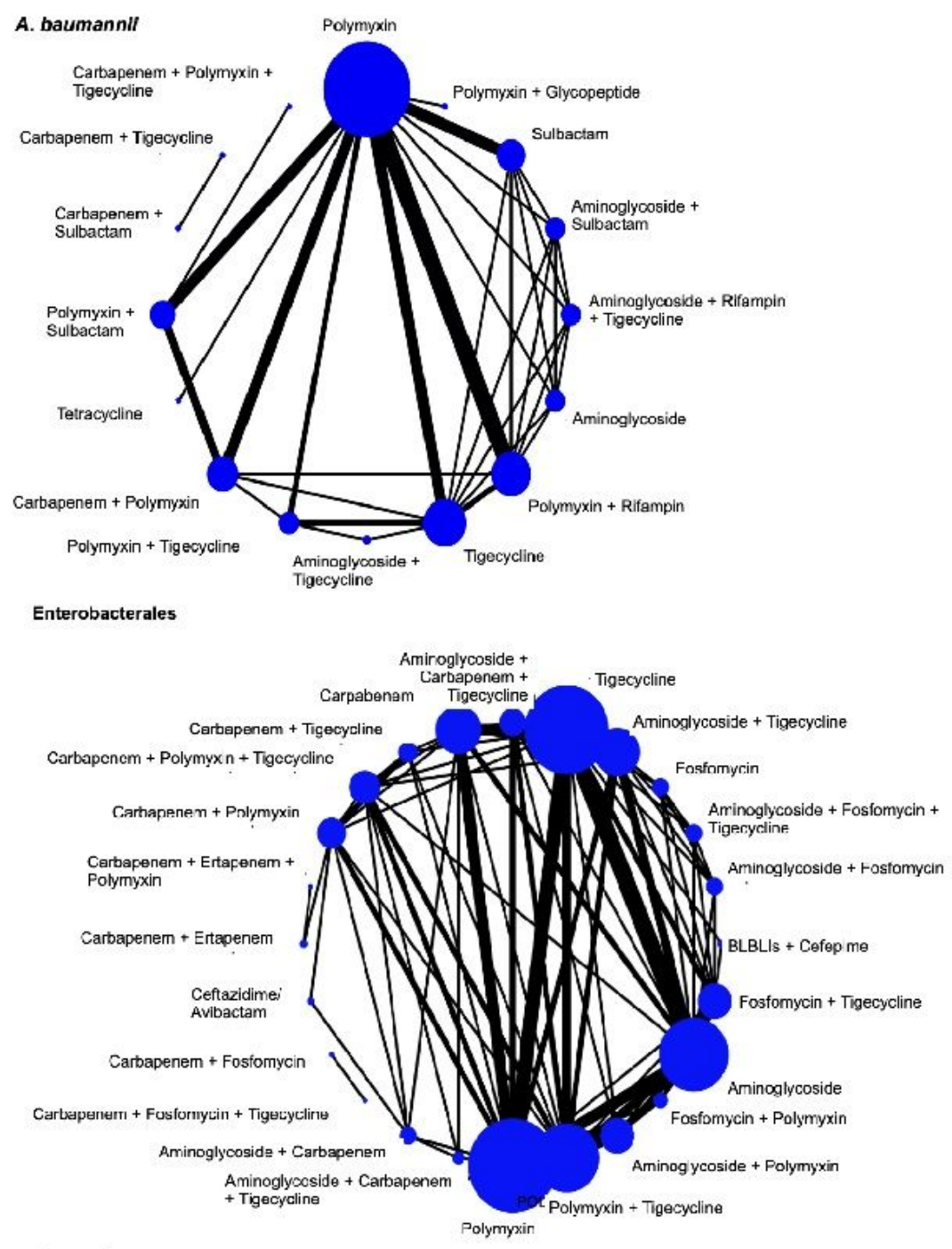

P. aeruginosa

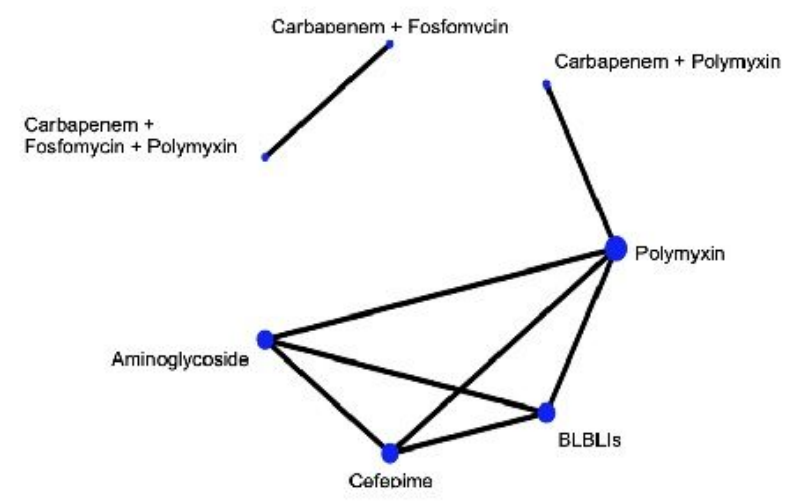

Figure 2

Network geometry of outcome mortality assessed for each bacterial phenotype. Legend: The nodes are weighted according to the number of patients included in the treatment regimen and edges are weighted according to the number of observations included in the respective comparison. For all bacterial phenotypes, the full closeness of the network is hampered by a variable number of disconnected components and the NMAs could not be run. BLBLIs: beta-lactam beta-lactam inhibitors. 


\section{Supplementary Files}

This is a list of supplementary files associated with this preprint. Click to download.

- COHERENCESupplementarySavoldi16022021.docx 